PROCEEDINGS OF THE

AMERICAN MATHEMATICAL SOCIETY

Volume 137, Number 8, August 2009, Pages 2661-2669

S 0002-9939(09)09875-X

Article electronically published on February 25, 2009

\title{
ON THE $L_{p}$ NORM OF THE RADEMACHER PROJECTION AND RELATED INEQUALITIES
}

\author{
LESŁAW SKRZYPEK
}

(Communicated by Nigel J. Kalton)

\begin{abstract}
The purpose of this paper is to find the exact norm of the Rademacher projection onto $\left\{r_{1}, r_{2}, r_{3}\right\}$. Namely, we prove

$$
\left\|R_{3}\right\|_{p}=\frac{\left(3^{p / q}+1\right)^{1 / p}\left(3^{q / p}+1\right)^{1 / q}}{4} .
$$

The same techniques also give the relative projection constant of $\operatorname{ker}\{1, \ldots, 1\}$ in $\ell_{p}^{n}$, that is,

$$
\lambda\left(\operatorname{ker}\{1, \ldots, 1\}, \ell_{p}^{n}\right)=\frac{\left((n-1)^{p / q}+1\right)^{1 / p}\left((n-1)^{q / p}+1\right)^{1 / q}}{n},
$$

for $n=2,3,4$. We discuss the relation of the above inequalities to the famous Khintchine and Clarkson inequalities. We conclude the paper by stating some conjectures that involve the geometry of the unit ball of $\ell_{p}^{n}$.
\end{abstract}

\section{INTRODUCTION}

The projection $P: X \rightarrow V$ is called minimal if it has the smallest possible norm, that is, if

$$
\|P\|=\lambda(V, X)=\inf \{\|Q\|: Q: X \rightarrow V \text { and } Q \text { is a projection }\} .
$$

Observe that any projection with norm one is automatically minimal though, in general, a given subspace will not be the range of a projection of norm 1 . The first problem is to find out whether a considered subspace is complemented, but even if that is the case, there could be no minimal projection (as the inf above does not have to be attained); for example, see [3. In many cases the existence of a minimal projection is known a priori (see [21, 11]), which is the case when the subspace is finite-dimensional or finite-codimensional. Even in such cases, minimal projections will be difficult to find. As a result of the dramatic evidence on the difficult nature of such problems, one may cite the fact that the minimal projections of $\mathcal{C}([0,1])$ onto the subspace of polynomials of degree $>2$ remain unknown. Let us mention several papers that characterize minimal projections in general settings [21, 6, 15, 17, 22, Still rarer is the situation in which the minimal projection is known to be unique. Even in $L_{p}(p \neq 2)$ spaces there are examples of minimal projections that are not unique (see [24]).

Received by the editors October 9, 2008 .

2000 Mathematics Subject Classification. Primary 41A65, 41A44, 42C10.

Key words and phrases. Minimal projections, Rademacher projection.

(C)2009 American Mathematical Society 
In two important cases we know minimal projections: the Fourier projection $F_{n}$ and the Rademacher projection $R_{n}$ are minimal in $L_{p}, 2,18$, , 5. The uniqueness of the Fourier projection in $C[0,1]$ and $L_{1}[0,1]$ has been settled in [7, 14]. In $L_{p}$ $(p \neq 2)$ the problem is open; the special case has been proved in [25].

Now we will discuss Rademacher projection. We will define the Rademacher functions, $r_{0}, r_{1}, \ldots$ by $r_{j}(t)=(-1)^{\left[2^{j} t\right]}$ for $0 \leq t \leq 1$, where [.] denotes the integer part of the argument. Since $r_{0} \equiv 1$ many authors consider only $r_{i}, i \geq 1\left(r_{i}\right.$ therefore being symmetric independent random variables). This is only a choice of convenience since

$$
\left\|a_{o} r_{0}+a_{1} r_{1}+\ldots+a_{n-1} r_{n-1}\right\|_{p}=\frac{1}{2}\left\|a_{o} r_{1}+a_{1} r_{2}+\ldots+a_{n-1} r_{n}\right\|_{p} .
$$

Put $\operatorname{Rad}_{n}=\operatorname{span}\left\{r_{1}, \ldots, r_{n}\right\}$ and $\widetilde{R a d}_{n}=\operatorname{span}\left\{r_{0}, \ldots, r_{n-1}\right\}$. The Rademacher projection $R_{n}$ (or $\widetilde{R}_{n}$ ) is an orthogonal projection onto $\operatorname{Rad}_{n}\left(\widetilde{\operatorname{Rad}}_{n}\right.$ correspondingly). Both spaces $\operatorname{Rad}_{n}$ and $\widetilde{R a d}_{n}$ are symmetric. As a result (see [16]) both Rademacher projections are unique minimal in $L_{p}[0,1](1<p<\infty)$. In $L_{1}[0,1]$ and $L_{\infty}[0,1]$, however, they are not unique minimal projections ([5, 26]).

The study of Rademacher functions came from the Khintchine inequality

$$
A_{p}\left\|\sum_{i=1}^{n} a_{i} r_{i}\right\|_{2} \leq\left\|\sum_{i=1}^{n} a_{i} r_{i}\right\|_{p} \leq B_{p}\left\|\sum_{i=1}^{n} a_{i} r_{i}\right\|_{2} .
$$

One can easily compute that

$$
\left\|\sum_{i=1}^{n} a_{i} r_{i}\right\|_{p}=\left(\text { Average }\left\{\left|\sum_{i=1}^{n} \epsilon_{i} a_{i}\right|^{p}: \epsilon_{i}= \pm 1\right\}\right)^{1 / p}=\left(E\left(\sum_{i=1}^{n} a_{i} r_{i}\right)^{p}\right)^{1 / p}
$$

and

$$
\left\|\sum_{i=1}^{n} a_{i} r_{i}\right\|_{2}=\left(\sum_{i=1}^{n}\left|a_{i}\right|^{2}\right)^{1 / 2}
$$

The problem of finding the best possible constants appearing in (1.3) has a long history and has finally been solved in ([10]). There are also generalizations of the Khintchine inequality to Banach spaces. The Rademacher projection can also be generalized and its norm has been compared to the Banach-Mazur distance (see [23] for exact formulations and more information). In [4] the norm of the Rademacher projection $R_{2}: L_{p} \rightarrow L_{q}$ has been computed. Paper [20] contains many interesting formulas involving integrals of Rademacher functions.

Unfortunately, $R_{3}$ may be the only Rademacher projection with a norm that can be succinctly expressed, though the author believes that, even in the general case, there could be an interesting formula to discover.

All of the above observations are also related to the relative projection constant of $\operatorname{ker}(1, \ldots, 1)$ in $\ell_{p}^{n}$. This is especially interesting due to the conjecture that $\operatorname{ker}(1, \ldots, 1)$ is the worst complemented hyperplane in $\ell_{p}^{n}(1<p<\infty)$. That is,

Conjecture 1.1. For any $f \in \ell_{q}^{n}, \lambda\left(\operatorname{ker} f, \ell_{p}^{n}\right) \leq \lambda\left(\operatorname{ker}(1, \ldots, 1), \ell_{p}^{n}\right)$.

It is worth mentioning that for $p=1$ and $p=\infty$ the relative projection constants $\lambda\left(\operatorname{ker} f, \ell_{1}^{n}\right)$ and $\lambda\left(\operatorname{ker} f, \ell_{\infty}^{n}\right)$ have been computed (see [3, 22]). 


\section{RESUlts}

The Rademacher projection is defined by $R_{n}=\sum_{i=1}^{n} r_{i} \otimes r_{i}$ and $\widetilde{R}_{n}=\sum_{i=0}^{n-1} r_{i} \otimes$ $r_{i}$. We can write the above projections as

$$
R_{n}(f)=\sum_{i=1}^{n}\left(\int_{0}^{1} r_{i}(t) f(t) d t\right) r_{i} \quad \text { and } \quad \widetilde{R}_{n}(f)=\sum_{i=0}^{n-1}\left(\int_{0}^{1} r_{i}(t) f(t) d t\right) r_{i}
$$

Our main goal is to find $\left\|R_{3}\right\|_{p}$ (we will see that actually $\left\|R_{3}\right\|_{p}=\left\|\widetilde{R}_{3}\right\|_{p}$ ).

Let $S_{2^{n}}$ denote the space with a basis of simple functions of intervals $I_{l, 2^{k}}=$ $\left(\frac{l}{2^{n}}, \frac{l+1}{2^{n}}\right)$, where $l \in\left\{0,1, \ldots, 2^{n}-1\right\}$.

Theorem 2.1. The $L_{p}$ norm of $\widetilde{R}_{n}$ is attained on $S_{2^{n-1}}$, and the $L_{p}$ norm of $R_{n}$ is attained on $S_{2^{n}}$.

Proof. We will prove the above for $\widetilde{R}_{n}$; for $R_{n}$ the proof is the same. We have $\widetilde{R a d} d_{n} \subset S_{2^{n-1}}$. Consider the projection

$$
T_{n}=\widetilde{R}_{n} / S_{2^{n-1}}: S_{2^{n-1}} \rightarrow \widetilde{\operatorname{Rad}}_{n} .
$$

$\left\|T_{n}\right\|_{p} \leq\left\|\widetilde{R}_{n}\right\|_{p}$. To prove the inequality in the opposite direction, consider the norm-one projection $L_{n-1}: L_{p}[0,1] \rightarrow S_{2^{n-1}}$ given by

$$
L_{n-1}(f)=\sum_{l=0}^{2^{n-1}-1} 2^{n-1}\left(\int_{I_{l, 2^{n-1}}} f(x) d x\right) \chi_{I_{l, 2^{n-1}}} .
$$

Furthermore, observe that $L_{n-1} / S_{2^{n-1}}=I d$, and since $r_{i} \in S_{2^{n-1}}$, we also have

$$
r_{i}(f)=r_{i}\left(L_{n-1} f\right) \text {. }
$$

Taking a norming point for $\widetilde{R}_{n}$, we have

$$
\begin{aligned}
\left\|\widetilde{R}_{n}\right\|_{p} & =\left\|\widetilde{R}_{n}(f)\right\|_{p}=\left\|\sum_{i=0}^{n-1} r_{i}(f) r_{i}\right\|_{p}=\left\|\sum_{i=0}^{n-1} r_{i}\left(L_{n-1} f\right) r_{i}\right\|_{p} \\
& =\left\|\widetilde{R}_{n}\left(L_{n-1} f\right)\right\|_{p}=\left\|T_{n}\left(L_{n-1} f\right)\right\|_{p} \leq\left\|T_{n}\right\|_{p} \cdot\left\|L_{n}\right\|_{p} \leq\left\|T_{n}\right\|_{p} .
\end{aligned}
$$

Hence, $\left\|\widetilde{R}_{n}\right\|_{p}=\left\|T_{n}\right\|_{p}$.

The following is easy to observe.

Remark 2.2. $\left\|R_{2}\right\|_{p}=\left\|\widetilde{R}_{2}\right\|_{p}=1$.

Our goal is to prove

Theorem 2.3. Consider $\widetilde{R}_{3}: L_{p}[0,1] \rightarrow\left\{r_{0}, r_{1}, r_{2}\right\}$. Then

$$
\left\|\widetilde{R}_{3}\right\|_{p}=\frac{\left(3^{p / q}+1\right)^{1 / p}\left(3^{q / p}+1\right)^{1 / q}}{4} .
$$

Proof. Since $\left(\widetilde{R}_{n}\right)^{*}=\widetilde{R}_{n}$ and $\left\|\left(\widetilde{R}_{n}\right)^{*}\right\|=\left\|\widetilde{R}_{n}\right\|$, we can assume that $p>2$. Put $w:=r_{1} r_{2}$. Then $r_{0}, r_{1}, r_{2}, w$ is a basis for $S_{4}$. Let $f=a_{0} r_{0}+a_{1} r_{1}+a_{2} r_{2}+a_{3} w$. Then it is easy to see that finding the norm of $R_{3}$ is equivalent to finding the best constant in the following inequality:

$$
\begin{aligned}
\left|a_{o}+a_{1}+a_{2}\right|^{p}+\left|a_{o}+a_{1}-a_{2}\right|^{p}+\mid & a_{o}-a_{1}+\left.a_{2}\right|^{p}+\left|a_{o}-a_{1}-a_{2}\right|^{p} \\
\leq C & \left(\left|a_{o}+a_{1}+a_{2}+a_{3}\right|^{p}+\left|a_{o}+a_{1}-a_{2}-a_{3}\right|^{p}\right. \\
& \left.+\left|a_{o}-a_{1}+a_{2}-a_{3}\right|^{p}+\left|a_{o}-a_{1}-a_{2}+a_{3}\right|^{p}\right),
\end{aligned}
$$


where $C^{1 / p}=\left\|R_{3}\right\|_{p}$. Making substitutions, $x_{1}=a_{0}+a_{1}+a_{2}, x_{2}=-a_{0}-a_{1}+a_{2}$, $x_{3}=-a_{0}+a_{1}-a_{2}, x_{4}=a_{0}-a_{1}-a_{2}$ and $d=a_{3}$, the above problem is reduced to

$$
C=\max \left\{\sum_{i=1}^{4}\left|x_{i}\right|^{p}: \sum_{i=1}^{4}\left|x_{i}+d\right|^{p}=1 \text { and } \sum_{i=1}^{4} x_{i}=0\right\}
$$

or equivalently,

$$
\lambda=\frac{1}{C}=\min \left\{\sum_{i=1}^{4}\left|x_{i}+d\right|^{p}: \sum_{i=1}^{4}\left|x_{i}\right|^{p}=1 \text { and } \sum_{i=1}^{4} x_{i}=0\right\} .
$$

We will write the above in the quotient form

$$
\lambda=\frac{1}{C}=\min \left\{\frac{\sum_{i=1}^{4}\left|x_{i}+d\right|^{p}}{\sum_{i=1}^{4}\left|x_{i}\right|^{p}}: \sum_{i=1}^{4} x_{i}=0\right\}=\min \left\{\frac{\sum_{i=1}^{4}\left|x_{i}+1\right|^{p}}{\sum_{i=1}^{4}\left|x_{i}\right|^{p}}: \sum_{i=1}^{4} x_{i}=0\right\} .
$$

Assume the above minimum is attained at $x=\left(x_{1}, x_{2}, x_{3}, x_{4}\right)$, where $\sum_{i=1}^{4} x_{i}=0$. Observe that $x \neq 0$, because if $x \rightarrow 0$, then $\frac{\sum_{i=1}^{4}\left|x_{i}+1\right|^{p}}{\sum_{i=1}^{4}\left|x_{i}\right|^{p}} \rightarrow \infty$. Take any $v=$ $\left(v_{1}, v_{2}, v_{3}, v_{4}\right)$ where $\sum_{i=1}^{4} v_{i}=0$ and consider the function

$$
g(\epsilon)=\frac{\sum_{i=1}^{4}\left|x_{i}+\epsilon v_{i}+1\right|^{p}}{\sum_{i=1}^{4}\left|x_{i}+\epsilon v_{i}\right|^{p}} .
$$

Since $p>2$ and $x=\left(x_{1}, x_{2}, x_{3}, x_{4}\right) \neq 0, g$ is a well-defined $C^{1}$ function around 0 . The function $g$ has a minimum at 0 . As a result, $g^{\prime}(0)=0$ and $g^{\prime \prime}(0) \geq 0$. From now on we will use the notation

$$
\langle x\rangle^{p}=|x|^{p} \operatorname{sign}(x) .
$$

Observe that $g=\frac{T}{S}$; therefore $g S=T$ and $g^{\prime} S+g S^{\prime}=T^{\prime}$. Using $g(0)=\lambda$ we obtain

$$
g^{\prime}(0)=\frac{p \sum_{i=1}^{4} v_{i}\left(\left\langle x_{i}+1\right\rangle^{p-1}-\lambda\left\langle x_{i}\right\rangle^{p-1}\right)}{\sum_{i=1}^{4}\left|x_{i}\right|^{p}}=0
$$

Taking a second derivative we get $g^{\prime \prime} S+2 g^{\prime} S^{\prime}+g S^{\prime \prime}=T^{\prime \prime}$. Using $g(0)=\lambda$ and $g^{\prime}(0)=0$ we get

$$
g^{\prime \prime}(0)=\frac{p(p-1) \sum_{i=1}^{4} v_{i}^{2}\left(\left|x_{i}+1\right|^{p-2}-\lambda\left|x_{i}\right|^{p-2}\right)}{\sum_{i=1}^{4}\left|x_{i}\right|^{p}} \geq 0 .
$$

Consider the following function:

$$
h(t)=\langle t+1\rangle^{p-1}-\lambda\langle t\rangle^{p-1} .
$$

Putting $v=(1,-1,0,0), v=(1,0,-1,0)$ and $v=(1,0,0,-1)$ in (2.13) gives us that for all $i, j$,

$$
h\left(x_{i}\right)=h\left(x_{j}\right)=A .
$$

Observe that $\lambda<1$. Taking the derivative of the function $h$, we can easily see that it has only two extreme points, a maximum at $t_{0}=-\frac{1}{1-\lambda^{1 / p-2}}$ and a minimum at $t_{1}=-\frac{1}{1+\lambda^{1 / p-2}}$. As a result, $h(t)=A$ can have a maximum of 3 different solutions. Therefore only 3 numbers among the $x_{i}$ can be different. We will prove that, in fact, only 2 numbers among the $x_{i}$ can be different. 
Assume for a contradiction that 3 numbers among the $x_{i}$ are different. Since $\sum_{i=1}^{4} x_{i}=0$ at least one of $x_{i}>0$. Thus, $h(t)=A$ has to have a positive solution. Observe now that $h(-1)=\lambda<1=h(0)$; therefore all negative solutions to $h(t)=$ $A$ have to be less than -1 . As a result, all the solutions of $h(t)=A$ have to be of the form

$$
-\alpha,-\beta, \gamma, \quad \text { where } \alpha>\beta>1, \gamma>0
$$

Observe that, until now, we have only used the first derivative of $g$. The above conditions guarantee us that $g$ is actually a $C^{\infty}$ function. There could be only one $-\beta$ among the $x_{i}$ (otherwise, since $h^{\prime}(-\beta)<0, g^{\prime \prime}(0)<0$, a contradiction to (2.14)). The possible choices for $x_{1}, x_{2}, x_{3}, x_{4}$ are now $-\alpha,-\alpha,-\beta, \gamma$ or $-\alpha,-\beta, \gamma, \gamma$. But since $\sum_{i=1}^{4} x_{i}=0$ in the first case, we have $\gamma=2 \alpha+\beta$, and secondly $\gamma=(\alpha+\beta) / 2$. In both cases

$$
\gamma \geq \beta
$$

Using (2.17), the equation $h(\gamma)=h(-\beta)$ gives

$$
\frac{(\gamma+1)^{p-1}+(\beta-1)^{p-1}}{\gamma^{p-1}+\beta^{p-1}}=\lambda<1
$$

On the other hand, since $\gamma \geq \beta$,

$$
(\gamma+1)^{p-1}-\gamma^{p-1} \geq(p-1) \gamma^{p-2} \geq(p-1) \beta^{p-2} \geq \beta^{p-1}-(\beta-1)^{p-1} .
$$

As a result,

$$
\frac{(\gamma+1)^{p-1}+(\beta-1)^{p-1}}{\gamma^{p-1}+\beta^{p-1}} \geq 1
$$

contrary to (2.19). Therefore we can have only 2 different numbers among the $x_{i}$, giving the possibilities $-a,-a, b, b$ and $-a, b, b, b(a>1, b>0)$. The first one is, in fact, not possible since it would lead to $b=a$, a contradiction. Hence $x_{1}, x_{2}, x_{3}, x_{4}$ has to be $-3 b, b, b, b(b>1 / 3)$. Plugging it into (2.10) and using $s=1 / b$ we get

$$
\lambda=\frac{1}{C}=\min _{b>1 / 3}\left\{\frac{3|b+1|^{p}+|3 b-1|^{p}}{3|b|^{p}+3^{p}|b|^{p}}\right\}=\frac{1}{3+3^{p}} \min _{0<s<3}\left\{3(1+s)^{p}+(3-s)^{p}\right\} \text {. }
$$

Standard calculations yield that the minimum is attained for $s=\frac{3-3^{q / p}}{1+3^{q / p}}$ and

$$
C=\frac{\left(3^{p / q}+1\right)\left(3^{q / p}+1\right)^{p / q}}{4^{p}}
$$

Theorem 2.4. $\left\|R_{3}\right\|_{p}=\left\|\widetilde{R}_{3}\right\|_{p}$.

Proof. Using the convexity of a function $f(x)=|x|^{p}$ we have $(2.24)$

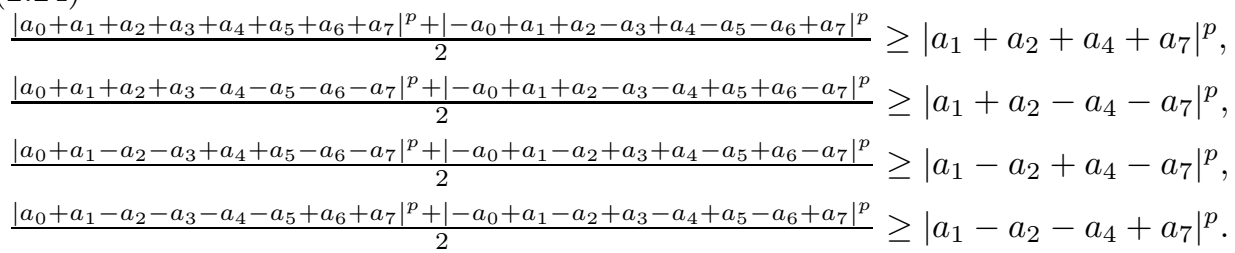


Using (1.2) and Theorem 2.3 we get

||$a_{1} r_{1}+a_{2} r_{2}+a_{4} r_{4}\left\|_{p}=2\right\| a_{1} r_{0}+a_{2} r_{1}+a_{3} r_{2}\left\|_{p} \leq 2\right\| \widetilde{R}_{3}\left\|_{p}\right\| a_{1} r_{0}+a_{2} r_{1}+a_{3} r_{2}+a_{7} w \|_{p}$, and applying equations (2.24), we get

$$
\left\|R_{3}\right\|_{p} \leq\left\|\widetilde{R}_{3}\right\|_{p}
$$

But since the above inequalities will become equalities when $a_{3}=a_{5}=a_{6}=0$, we will have equality.

From the proof of the above Theorem 2.3 we can deduce that

Corollary 2.5. For any $a_{0}, a_{1}, a_{2}, a_{3} \in \mathbb{R}$,

$$
\begin{gathered}
\left|a_{o}+a_{1}+a_{2}\right|^{p}+\left|a_{0}+a_{1}-a_{2}\right|^{p}+\left|a_{0}-a_{1}+a_{2}\right|^{p}+\left|a_{0}-a_{1}-a_{2}\right|^{p} \\
\leq C_{p}\left(\left|a_{0}+a_{1}+a_{2}+a_{3}\right|^{p}+\left|a_{0}+a_{1}-a_{2}-a_{3}\right|^{p}\right. \\
\left.+\left|a_{0}-a_{1}+a_{2}-a_{3}\right|^{p}+\left|a_{0}-a_{1}-a_{2}+a_{3}\right|^{p}\right),
\end{gathered}
$$

where $C_{p}=\frac{\left(3^{p / q}+1\right)\left(3^{q / p}+1\right)^{p / q}}{4^{p}}$ is the best possible constant.

There are several inequalities known that contain the left part of the above inequality. The following inequality was used to obtain important results on sums of independent random variables.

Theorem 2.6 ([15], Lemma 5). For any $a_{0}, a_{1}, a_{2} \in \mathbb{R}$,

$$
\begin{aligned}
\left|a_{0}+a_{1}+a_{2}\right|^{p}+\left|a_{0}+a_{1}-a_{2}\right|^{p}+\left|a_{0}-a_{1}+a_{2}\right|^{p}+\left|a_{0}-a_{1}-a_{2}\right|^{p} \\
\leq \frac{\left(\left|a_{0}+a_{1}\right|^{p}+\left|a_{0}-a_{1}\right|^{p}\right)\left(\left|a_{0}+a_{2}\right|^{p}+\left|a_{0}-a_{2}\right|^{p}\right)}{\left|a_{0}\right|^{p}} .
\end{aligned}
$$

The left side of (2.27) can be viewed as

$$
4 * \text { Average }\left\{\left|\epsilon_{0} a_{0}+\epsilon_{1} a_{1}+\epsilon_{2} a_{2}\right|^{p}: \epsilon_{i}= \pm 1\right\} ;
$$

therefore it is related to Khintchine-type Inequalities. The following inequality can be deduced from it.

Theorem 2.7 (9], Proposition 2.1; 13], Corollary 3). For any $a_{0}, a_{1}, a_{2} \in \mathbb{R}$ and $p>2$,

$$
\begin{gathered}
\left|a_{0}+a_{1}+a_{2}\right|^{p}+\left|a_{0}+a_{1}-a_{2}\right|^{p}+\left|a_{0}-a_{1}+a_{2}\right|^{p}+\left|a_{0}-a_{1}-a_{2}\right|^{p} \\
\leq\left(3^{p / q}+1\right)\left(\left|a_{0}\right|^{p}+\left|a_{1}\right|^{p}+\left|a_{2}\right|^{p}\right) .
\end{gathered}
$$

The above results are also connected to the famous Clarkson Inequalities (see [19, 12 for generalizations).

Theorem 2.8 (Clarkson Inequality [8]). For any $a_{0}, a_{1} \in \mathbb{R}$ and $p>2$,

$$
\left|a_{0}+a_{1}\right|^{p}+\left|a_{0}-a_{1}\right|^{p} \leq 2^{p / q}\left(\left|a_{0}\right|^{p}+\left|a_{1}\right|^{p}\right) .
$$

We will now compute the relative projection constants of $\operatorname{ker}(1, \ldots, 1)$ in $\ell_{p}^{n}$. We will need the following.

Theorem 2.9. The following holds:

$$
\min \left\{\frac{\sum_{i=1}^{n}\left|a_{i}+d\right|^{p}}{\sum_{i=1}^{n}\left|a_{i}\right|^{p}}: \sum_{i=1}^{n} a_{i}=0\right\}=\frac{n^{p}}{\left((n-1)^{p / q}+1\right)\left((n-1)^{q / p}+1\right)^{p / q}} .
$$


Proof. The case $n=2$ is trivial, and the case $n=4$ follows from Theorem 2.3. If $n=3$, the proof goes the same way as in Theorem 2.3. since $x_{1}+x_{2}+x_{3}=0$ gives $\alpha+\beta+\gamma=0$ and then $\gamma=\alpha+\beta>\beta$ and the rest follows from the same reasoning as in the $n=4$ case. The general case can be handled in a similar way as in $n=4$. The proof becomes extremely tedious and depends on the consideration of many cases. The author does not know a satisfactory solution in the general case.

The following is an easy consequence of the above.

Theorem 2.10. Consider $P=I d-\frac{1}{n} 1 \otimes 1: \ell_{p}^{n} \rightarrow$ ker 1 . Then for $n=2,3,4$ and $1 \leq p \leq \infty$,

$$
\|P\|_{p}=\frac{\left((n-1)^{p / q}+1\right)^{1 / p}\left((n-1)^{q / p}+1\right)^{1 / q}}{n} .
$$

Moreover, for $1<p<\infty, p \neq 2$, if $x \in S\left(\ell_{p}^{n}\right)$ is a norming point for $P$, then $x=\left(x_{1}, \ldots, x_{n}\right)= \pm\left(u_{\pi(1)}, \ldots, u_{\pi(n)}\right)$, where $\pi$ is any permutation and

$$
u=\frac{1}{\left((n-1)+(n-1)^{q}\right)^{1 / p}}\left(-(n-1)^{q / p}, 1, \ldots, 1\right) .
$$

Proof. We have

$$
P x=x-\frac{1}{n}\left(\sum_{i=1}^{n} x_{i}\right)(1, \ldots, 1) .
$$

Taking $a_{i}=x_{i}-\frac{1}{n}\left(\sum_{i=1}^{n} x_{i}\right)$ and $d=\frac{1}{n}\left(\sum_{i=1}^{n} x_{i}\right)$ and applying Theorem 2.9, we get the result.

The above projection is minimal [16. Interestingly, because of the form of the norming points of $P$, we can see that it is not a minimal projection with respect to the numerical radius (see [1] for details). As a result of the above theorem, we automatically get

Theorem 2.11. For $n=2,3,4$ and $1 \leq p \leq \infty$, the relative projection constant is

$$
\lambda\left(\operatorname{ker}(1,1, \ldots, 1), \ell_{p}^{n}\right)=\frac{\left((n-1)^{p / q}+1\right)^{1 / p}\left((n-1)^{q / p}+1\right)^{1 / q}}{n} .
$$

We will conclude this article by stating two conjectures that relate to the geometry of the $\ell_{p}^{n}$ unit ball.

Conjecture 2.12. Assume $n \geq 3$ and $1<p<\infty, p \neq 2$. Let $S$ be a unit sphere in $\ell_{p}^{n}$ and let $S_{d}$ be the shift of $S$ by the vector $(d, \ldots, d)$. Consider the ring $R=$ $S \cap \operatorname{ker}(1, \ldots, 1)$. Now we can shrink or enlarge $S_{d}$ in such a way that it will be tangent to $R$ and $R$ will be outside of $S_{d}$. Then the points where $R$ and $c \cdot S_{d}$ meet have exactly $n-1$ coordinates equal. As a result we have exactly $2 n$ points of tangency of $c \cdot S_{d}$ and $R$.

The above conjecture is equivalent to showing that

$$
\min \left\{\sum_{i=1}^{n}\left|x_{i}+d\right|^{p}: \sum_{i=1}^{n}\left|x_{i}\right|^{p}=1 \text { and } \sum_{i=1}^{n} x_{i}=0\right\}
$$

is attained at points that have exactly $n-1$ coordinates equal. This is also equivalent to Theorem 2.9 (we gave a satisfactory proof of it for $n=3,4$ ). 
Conjecture 2.13. Assume $n \geq 3$ and $1<p<\infty, p \neq 2$. Let $S$ be a unit sphere in $\ell_{p}^{n}$ and let $S_{d}$ be the shift of $S$ by the vector $(d, \ldots, d)$. Consider the ring $R=$ $S \cap \operatorname{ker}(1, \ldots, 1)$. Now we can shrink or enlarge $S_{d}$ in such a way that it will be tangent to $R$ and $R$ will be inside of $S_{d}$. Then the coordinates of the points where $R$ and $c \cdot S_{d}$ meet are such that $n-2$ of them are equal and also the remaining 2 are equal to each other. As a result we have exactly $n(n-1)$ points of tangency of $c \cdot S_{d}$ and $R$.

The above conjecture is equivalent to showing that

$$
\max \left\{\sum_{i=1}^{n}\left|x_{i}+d\right|^{p}: \sum_{i=1}^{n}\left|x_{i}\right|^{p}=1 \text { and } \sum_{i=1}^{n} x_{i}=0\right\}
$$

is attained at points in which the coordinates are such that $n-2$ of them are equal and also the remaining 2 are equal to each other.

The dilemma in (2.37) and (2.38) is that both functions have several local minima and maxima (for instance, numerical calculations show that (2.38) has a local maximum with three different numbers among $x_{1}, \ldots, x_{n}$ ), so one has to be very careful when trying to find any numerical solutions to the above.

\section{REFERENCES}

1. A. G. Aksoy and B. L. Chalmers, Minimal numerical-radius extensions of operators, Proc. Amer. Math. Soc. 135 (2007), pp. 1039-1050 (electronic). MR.2262904 (2007j:46002)

2. D. L. Berman, On the impossibility of constructing a linear polynomial operator furnishing an approximation of the order of the best approximation, Dokl. Akad. Nauk SSSR 120 (1958), pp. 1175-1177. MR0098941 (20:5387)

3. J. Blatter and E. W. Cheney, Minimal projections on hyperplanes in sequence spaces, Ann. Mat. Pura Appl. (4) 101 (1974), pp. 215-227. MR0358179 (50:10644)

4. B. L. Chalmers and F. T. Metcalf, Critical points of the $n$-dimensional Khintchine ratio and Rademacher projection norms, Approximation theory VI, Vol. I (College Station, TX, 1989), Academic Press, Boston, MA, 1989, pp. 125-128. MR1090980 (91m:41056)

5. - The determination of minimal projections and extensions in $L^{1}$, Trans. Amer. Math. Soc. 329 (1992), pp. 289-305. MR.1034660 (92e:41017)

6. - A characterization and equations for minimal projections and extensions, J. Operator Theory 32 (1994), pp. 31-46. MR.1332442 (96c:46014)

7. E. W. Cheney, C. R. Hobby, P. D. Morris, F. Schurer, and D. E. Wulbert, On the minimal property of the Fourier projection, Trans. Amer. Math. Soc. 143 (1969), pp. 249-258. MR0256044 (41:704)

8. J. A. Clarkson, Uniformly convex spaces, Trans. Amer. Math. Soc. 40 (1936), pp. 396-414. MR 1501880

9. T. Figiel, T. Iwaniec, and A. Pełczyński, Computing norms and critical exponents of some operators in $L^{p}$-spaces, Studia Math. 79 (1984), pp. 227-274. MR781720 (86m:46018)

10. U. Haagerup, The best constants in the Khintchine inequality, Studia Math. 70 (1981), pp. 231-283 (1982). MR654838 (83m:60031)

11. J. R. Isbell and Z. Semadeni, Projection constants and spaces of continuous functions, Trans. Amer. Math. Soc. 107 (1963), pp. 38-48. MR0146649 (26:4169)

12. M. Kato, Generalized Clarkson's inequalities and the norms of the Littlewood matrices, Math. Nachr. 114 (1983), pp. 163-170. MR745054 (85i:47011)

13. R. Komorowski, On the best possible constants in the Khintchine inequality for $p \geq 3$, Bull. London Math. Soc. 20 (1988), pp. 73-75. MR916079(89e:60037)

14. P. V. Lambert, On the minimum norm property of the Fourier projection in $L^{1}$-spaces, Bull. Soc. Math. Belg. 21 (1969), pp. 370-391. MR0273293 (42:8173)

15. R. Latała, Estimation of moments of sums of independent real random variables, Ann. Probab. 25 (1997), pp. 1502-1513. MR1457628 (98h:60021)

16. G. Lewicki and L. Skrzypek, Chalmers-Metcalf operator and uniqueness of minimal projections, J. Approx. Theory 148 (2007), pp. 71-91. MR2356576 
17. in analysis, Walter de Gruyter, Berlin, 2007, pp. 375-389. MR2374721

18. S. M. Lozinskǐ̌, On a class of linear operations, Doklady Akad. Nauk SSSR (N. S.) 61 (1948), pp. 193-196. MR.0026699 (10:188c)

19. L. Maligranda and L. E. Persson, On Clarkson's inequalities and interpolation, Math. Nachr. 155 (1992), pp. 187-197. MR.1231264 (94h:46113)

20. F. T. Metcalf, An integral identity for the Rademacher functions, J. Math. Anal. Appl. 189 (1995), pp. 393-408. MR1312052 (95k:42050)

21. P. D. Morris and E. W. Cheney, On the existence and characterization of minimal projections, J. Reine Angew. Math. 270 (1974), pp. 61-76. MR0358188 (50:10653)

22. W. Odyniec and G. Lewicki, Minimal projections in Banach spaces. Problems of existence and uniqueness and their application. Lecture Notes in Math., vol. 1449, Springer-Verlag, Berlin, 1990. MR.1079547 (92a:41021)

23. G. Pisier, The volume of convex bodies and Banach space geometry, Cambridge Tracts in Math., vol. 94, Cambridge University Press, Cambridge, 1989. MR.1036275 (91d:52005)

24. B. Shekhtman and L. Skrzypek, On the non-uniqueness of minimal projection in $L_{p}$ spaces, J. Approx. Theory. Article in press. doi:10.1016/j.jat.2008.08.006

25. - Norming points and unique minimality of orthogonal projections, Abstr. Appl. Anal. (2006), Art. ID 42305, 17 pp. MR2211664 (2006k:46043)

26. L. Skrzypek, On the non-uniqueness of minimal projections in $L_{1}$, preprint.

Department of Mathematics, University of South Florida, 4202 E. Fowler Avenue, PHY 114, TAMPA, FlORIDA 33620-5700

E-mail address: skrzypek@math.usf.edu 